\title{
COMPACTNESS PROPERTIES OF AN OPERATOR WHICH IMPLY THAT IT IS AN INTEGRAL OPERATOR BY
}

\author{
A. R. SCHEP
}

\begin{abstract}
In this paper we study necessary and (or) sufficient conditions on a given operator to be an integral operator. In particular we give another proof of a characterization of integral operators due to $\mathrm{W}$. Schachermayer.
\end{abstract}

Introduction. This paper consists of two parts. In the first part we investigate sufficient conditions for operators, with domain $L_{1}(Y, \nu)$ or range in $L_{\infty}(X, \mu)$, to be integral operators. As a special case we get that weakly compact operators from $L_{1}(Y, \nu)$ into $L_{1}(X, \mu)$ are kernel operators and that they constitute an order ideal in $\mathcal{L}\left(L_{1}, L_{1}\right)$. The corresponding results for weakly compact operators from $L_{\infty}(Y, \nu)$ into $L_{\infty}(X, \mu)$ also hold. In the second part we give an elementary proof of a generalization of the following result due to $\mathrm{W}$. Schachermayer. If $T$ is a linear operator from $L_{2}(Y, \nu)$ into $L_{2}(X, \mu)$, then $T$ is an integral operator if and only if $T$ maps order intervals into equimeasurable sets. We shall indicate the relation between this result and results of A. Grothendieck and of R. J. Nagel and U. Schlotterbeck.

1. Preliminaries. $(Y, \Sigma, \nu)$ and $(X, \Lambda, \mu)$ will always denote in this paper $\sigma$-finite measure spaces to which the Carathéodory extension procedure has been applied. The set of all realvalued $\mu$-measurable functions will be denoted by $M(X, \mu)$, where functions equal a.e. are identified. A linear subspace $M$ of $M(X, \mu)$ is called an (order) ideal of measurable functions, whenever it follows from $f \in M$ and $g \in M(X, \mu)$ with $|g| \leqslant|f|$ that $g \in M$. An order ideal $L \subset M(X, \mu)$ provided with an absolute and monotone norm $\rho$ is called a normed function space and denoted by $L_{\rho}$. If the normed function space is norm complete it is called a Banach function space [12, Chapter 15]. Let $L$ and $M$ be ideals of measurable functions in $M(Y, \nu)$ and $M(X, \mu)$, respectively. Then a linear operator $T: L \rightarrow M$ is called an integral operator if there exists a $(\mu \times \nu)$-measurable $T(x, y) \in M(X \times Y, \mu \times \nu)$ such that, for every $f \in L, T f(x)=\int T(x, y) f(y) d \nu(y)$ holds almost everywhere on $X$. An order bounded (absolutely bounded) integral operator $T$ from $L$ into $M$ is here called an absolute integral operator. If $T$ is an absolute integral operator, then $|T(x, y)|$ is also the kernel of an operator from $L$ into $M$.

If $L_{\rho}$ is a Banach function space, then $L_{\rho}^{\prime}$ denotes the first associate space and $\rho^{\prime}$ the first associate norm. If $\rho$ has the weak Fatou property, then $L_{\rho}$ and $L_{\rho}^{\prime \prime}$ coincide

Received by the editors October 18, 1979 and, in revised form, April 7, 1980.

1980 Mathematics Subject Classification. Primary 47G05, 45P05; Secondary 47B38, 47B55. 
as sets and $\rho$ and $\rho^{\prime \prime}$ are equivalent norms (see [12]). The following theorem is due to W. A. J. Luxemburg [5].

THEOREM 1.1. Let $L_{\rho}=L_{\rho}(X, \mu)$ be a Banach function space and let $H \subset L_{\rho}$. If $H$ is $\sigma\left(L_{\rho}, L_{\rho}^{\prime}\right)$ relatively compact, then the following conditions hold.

(i) For all $g \in L_{\rho}^{\prime}$ we have $\sup \left(\int_{X}|f g| d \mu: f \in H\right)<\infty$.

(ii) For all $\varepsilon>0$ and all $g \in L_{\rho}^{\prime}$ there exists $\delta>0$ such that $A \in \Lambda$ with $\mu(A)<\delta$ implies that $\sup \left(\int_{A}|g f| d \mu: f \in H\right) \leqslant \varepsilon$.

(iii) For all $\varepsilon>0$ and all $g \in L_{\rho}^{\prime}$ there exists $X_{0} \subset X$ with $\mu\left(X_{0}\right)<\infty$ such that for the complement $X_{0}^{c}=X \backslash X_{0}$ we have $\sup \left(\int_{X_{0}^{c}}|f g| d \mu: f \in H\right)<\varepsilon$.

Conversely, if $\rho$ has the weak Fatou property, then (i)-(iii) imply that $H$ is $\sigma\left(L_{\rho}, L_{\rho}^{\prime}\right)$ relatively compact.

REMARKS. 1. The above theorem is a generalization of the well-known DunfordPettis theorem on relatively weakly compact subsets of $L_{1}(\mu)$ and can be reduced to it by observing that $g H=\{g h: h \in H\}$ is a relatively weakly compact subset of $L_{1}(\mu)$ in case $g \in L_{\rho}^{\prime}$ and $H$ is $\sigma\left(L_{\rho}, L_{\rho}^{\prime}\right)$ relatively compact.

2. It is not difficult to show that (ii) and (iii) together are equivalent with:

If $g_{n} \in L_{\rho}^{\prime}$ and $g_{n} \downarrow 0$ a.e. then $\sup \left(\int\left|f g_{n}\right| d \mu: f \in H\right) \downarrow 0$.

3. If $\rho$ is weakly Fatou, then it follows from the above theorem that in case $H \subset L_{\rho}$ is $\sigma\left(L_{\rho}, L_{\rho}^{\prime}\right)$ relatively compact, then also the solid hull $\left\{g \in L_{\rho}:|g|<h\right.$, $h \in H\}$ is $\sigma\left(L_{\rho}, L_{\rho}^{\prime}\right)$ relatively compact.

2. Operators with domain $L_{1}(Y, \nu)$ or range in $L_{\infty}(X, \mu)$. We first recall the following fundamental result (see [10] for an elementary proof and references).

Theorem 2.1 (A. V. Buhvalov). Let $L$ and $M$ be ideals of measurable functions in $M(Y, \nu)$ and $M(X, \mu)$, respectively. Then a linear operator $T: L \rightarrow M$ is an integral operator if and only if it follows from $0 \leqslant u_{n} \leqslant u$ in $L$ and $u_{n} \stackrel{*}{\rightarrow} 0$ that $T u_{n}(x) \rightarrow 0$ a.e.

The notation $u_{n} \stackrel{*}{\rightarrow} 0$ means here that every subsequence of $\left\{u_{n}\right\}$ contains a subsequence converging to zero a.e.

THeOREM 2.2. Let $L_{\rho}$ be a Banach function space and let $T: L_{1}(Y, \nu) \rightarrow L_{\rho}(X, \mu)$ be a norm bounded $\sigma\left(L_{\rho}, L_{\rho}^{\prime}\right)$ compact operator. Then $T$ is an integral operator.

Proof. Let $X=\cup_{n=1}^{\infty} X_{n}$ such that $\mu\left(X_{n}\right)<\infty, X_{n} \cap X_{m}=\varnothing$ for $n \neq m$ and $\chi_{X_{n}} \in L_{\rho}^{\prime}$ for all $n$. Let $P_{n} f=f \chi_{X_{n}}$. Then $P_{n} \circ T$ is a $\sigma\left(L_{\rho}\left(X_{n}, \mu\right), L_{\rho}^{\prime}\left(X_{n}, \mu\right)\right)$ compact operator from $L_{1}(Y, \nu)$ into $L_{\rho}\left(X_{n}, \mu\right)$. If $P_{n} \circ T$ is an integral operator for all $n$, then $T$ is also an integral operator. Therefore we may assume in the proof that $\chi_{X} \in L_{\rho}^{\prime}$ and $\mu(X)<\infty$. Denote by $T^{\prime}$ the restriction of the adjoint operator to $L_{\rho}^{\prime}$. We first prove that $T^{\prime}$ is a kernel operator from $L_{\rho}^{\prime}$ into $L_{\infty}(Y, \nu)$. Let $0<g_{n}<g$ $\in L_{\rho}^{\prime}$ and assume $g_{n} \stackrel{*}{\rightarrow} 0$. We shall prove that $\left\|T^{\prime} g_{n}\right\|_{\infty} \rightarrow 0$. Let $\varepsilon>0$ be given. Then, by Theorem 1.1, there exists $\delta>0$ such that $\mu(A)<\delta$ implies

$$
\sup \left(\int_{A} g(x)|T f(x)| d \mu(x):\|f\|_{1} \leqslant 1\right)<\frac{\varepsilon}{2} .
$$


Let $\eta=\varepsilon / 2\left\|T^{\prime}\right\| \rho^{\prime}\left(\chi_{X}\right)$ and put $A_{n}=\left\{x: g_{n}(x)>\eta\right\}$. It follows from $g_{n} \stackrel{*}{\rightarrow} 0$ that $\mu\left(A_{n}\right) \rightarrow 0$. Let $N$ be such that $\mu\left(A_{n}\right) \leqslant \delta$ for all $n>N$. Then, for all $n>N$, we have

$$
\sup \left(\int_{A_{n}} g(x)|T f(x)| d \mu(x):\|f\|_{1}<1\right)<\frac{\varepsilon}{2}
$$

so also

$$
\sup \left(\left|\int_{A_{n}} g_{n}(x) T f(x) d \mu(x)\right|:\|f\|_{1} \leqslant 1\right)<\frac{\varepsilon}{2}
$$

for $n \geqslant N$. From this we conclude that $\left\|T^{\prime}\left(g_{n} \chi_{A_{n}}\right)\right\|_{\infty}<\varepsilon / 2$ for $n>N$. Hence

$$
\begin{aligned}
\left\|T^{\prime} g_{n}\right\|_{\infty} & \leqslant\left\|T^{\prime}\left(g_{n} \chi_{A_{n}}\right)\right\|_{\infty}+\left\|T^{\prime}\left(g_{n} \chi_{A_{n}}\right)\right\|_{\infty} \\
& \leqslant \varepsilon / 2+\left\|T^{\prime}\right\| \cdot \rho^{\prime}\left(g_{n} \chi_{A_{n}^{c}}\right) \leqslant \varepsilon / 2+\left\|T^{\prime}\right\| \cdot \eta \cdot \rho^{\prime}\left(\chi_{X}\right)=\varepsilon
\end{aligned}
$$

for all $n \geqslant N$. It follows that $\left\|T^{\prime} g_{n}\right\|_{\infty} \rightarrow 0$, so certainly $T^{\prime} g_{n}(x) \rightarrow 0$ a.e. Hence $T^{\prime}$ is an integral operator from $L_{\rho}^{\prime}$ into $L_{\infty}(Y, \nu)$. Since, however, every norm bounded operator into $L_{\infty}(Y, \nu)$ is order bounded, we conclude that $T$ is an absolute integral operator. This implies that $T^{\prime \prime}$ is an absolute integral operator into $L_{\rho}^{\prime \prime}(X, \mu)$, so $T$ is an integral operator from $L_{1}(Y, \nu)$ into $L_{\rho}(X, \mu)$ (the modulus of its kernel defines an operator from $L_{1}$ into $L_{\rho}^{\prime \prime}$ and need not map $L_{1}$ into $L_{\rho}$ ).

Remarks. (i) If we add to the hypotheses in the above theorem that $\rho$ has the weak Fatou property, then $T$ is an absolute integral operator from $L_{1}$ into $L_{\rho}$.

(ii) If $T$ is as in the above theorem and has kernel $T(x, y)$, then for $\nu$-a.e. $y \in Y$ we have $T_{y}(x)=T(x, y) \in L_{\rho}^{\prime \prime}(X, \nu)$. This follows from the observation that $\left|T^{\prime} f(y)\right| \leqslant\left\|T^{\prime}\right\| \rho^{\prime}(f) \nu$-a.e. and the methods used in [11].

Corollary 2.3. If $T: L_{1}(Y, \nu) \rightarrow L_{1}(X, \mu)$ is weakly compact, then $T$ is an absolute integral operator.

We shall now investigate the order structure of the set of $\sigma\left(L_{\rho}, L_{\rho}^{\prime}\right)$ compact kernel operators from $L_{1}(Y, \nu)$ into $L_{\rho}(X, \mu)$. We first notice that for a proof that a positive operator $T$ from $L_{1}(Y, \nu)$ into $L_{\rho}(X, \mu)$ is $\sigma\left(L_{\rho}, L_{\rho}^{\prime}\right)$ compact, it suffices to show that $\left\{T f: 0 \leqslant f \in L_{1}(Y, \nu),\|f\|_{1} \leqslant 1\right\}$ is $\sigma\left(L_{\rho}, L_{\rho}^{\prime}\right)$ relatively compact. We also notice that in case $\rho$ has the weak Fatou property, then the space $\mathcal{L}_{b}\left(L_{1}, L_{\rho}\right)$ of all order bounded operators is equal to the space $\mathcal{L}\left(L_{1}, L_{\rho}\right)$ of all norm bounded operators.

THEOREM 2.4. Let $L_{\rho}$ be a Banach function space and assume that $\rho$ has the weak Fatou property. Then the set of all $\sigma\left(L_{\rho}, L_{\rho}^{\prime}\right)$ compact operators from $L_{1}(Y, \nu)$ into $L_{\rho}(X, \mu)$ is an order ideal in $\mathcal{L}_{b}\left(L_{1}, L_{\rho}\right)$.

Proof. Assume $T: L_{1} \rightarrow L_{\rho}$ is $\sigma\left(L_{\rho}, L_{\rho}^{\prime}\right)$ compact. We show that the set $\left\{|T| f: 0 \leqslant f \in L_{1},\|f\|_{1} \leqslant 1\right\}$ satisfies the conditions of Theorem 1.1. It clearly satisfies condition (i), since it is a norm bounded set. To prove (ii), let $0 \leqslant g \in L_{\rho}^{\prime}$ and $\varepsilon>0$ be given. Then there exists $\delta>0$ such that $\mu(A)<\delta$ implies that

$$
\sup \left(\int_{A}|T f| g d \mu: f \in L_{1},\|f\|_{1}<1\right)<\varepsilon .
$$


Let $0 \leqslant u \in L_{1}$ with $\|u\|_{1} \leqslant 1$. Then we have

$$
|T| u=\sup \left(\sum_{i=1}^{n}\left|T u_{i}\right|: u=\sum_{1}^{n} u_{i}, 0 \leqslant u_{i} \in L_{1}\right)
$$

and we observe that the set, of which the supremum is taken, is directed upwards. Hence we have, for all $A \in \Lambda$,

$$
\int_{A}|T| u \cdot g d \mu=\sup \left(\sum_{1}^{n} \int_{A}\left|T u_{i}\right| g d \mu: u=\sum_{1}^{n} u_{i}, 0 \leqslant u_{i} \in L_{1}\right) .
$$

Let $\mu(A) \leqslant \delta$ and let $0 \leqslant u_{i} \in L_{1}$ with $u=\sum_{1}^{n} u_{i}$. Then it follows from (1) that $\int_{A}\left|T u_{i}\right| g d \mu \leqslant \varepsilon\left\|u_{i}\right\|_{1}$ for $i=1,2, \ldots, n$, so

$$
\sum_{1}^{n} \int_{A}\left|T u_{i}\right| g d \mu \leqslant \varepsilon \sum_{1}^{n}\left\|u_{i}\right\|_{1}=\varepsilon\|u\|_{1} \leqslant \varepsilon .
$$

We conclude that for $A \in \Lambda$, with $\mu(A) \leqslant \delta$,

$$
\sup \left(\int_{A}|T| u \cdot g d \mu: u \in L_{1}^{+},\|u\|_{1} \leqslant 1\right) \leqslant \varepsilon,
$$

i.e., condition (ii) of Theorem 1.1 is satisfied. The proof of condition (iii) is completely similar and therefore omitted. It follows now that $|T|$ is $\sigma\left(L_{\rho}, L_{\rho}^{\prime}\right)$ compact. If now $0 \leqslant S \leqslant T$ and $T$ is $\sigma\left(L_{\rho}, L_{\rho}^{\prime}\right)$ compact, then it follows immediately from Theorem 1.1 that $S$ is $\sigma\left(L_{\rho}, L_{\rho}^{\prime}\right)$ compact. Hence the proof of the theorem is complete.

EXAMPLE. Let $X=Y=[0,1]$ and $\mu=\nu$ be Lebesgue measure. Let $g_{n}(x)$ be the characteristic function of the interval $\left(1 / 2^{n+1}, 1 / 2^{n}\right)$ and let $\phi_{n}$ be the $n$th Rademacher function. We define $T: L_{1}(Y, \nu) \rightarrow L_{\infty}(X, \mu)$ by means of

$$
T f(x)=\sum_{n=0}^{\infty}\left(\int_{0}^{1} f(y) \phi_{n}(y) d y\right) g_{n}(x)
$$

Then $T$ is not weakly compact, but $T$ is $\sigma\left(L_{\infty}, L_{1}\right)$ compact. Since $|T|$ is a rank one operator, it follows that the above theorem is false for weakly compact operators.

We now dualize the above results. The following theorem singles out the right notion of duality for $\sigma\left(L_{\rho}, L_{\rho}^{\prime}\right)$ compact operators from $L_{1}$ into $L_{\rho}$.

THEOREM 2.5. Let $L_{\rho}=L_{\rho}(Y, \nu)$ be a Banach function space and let $T: L_{\rho}(Y, \nu)$ $\rightarrow L_{\infty}(X, \mu)$ be a bounded linear operator. Then the following are equivalent.

(i) $T$ is order continuous and $T[0, g]$ is $\sigma\left(L_{\infty}, L_{\infty}^{*}\right)$ relatively compact for all $0<g \in L_{\rho}$.

(ii) For all $0 \leqslant g_{n} \leqslant g \in L_{\rho}$ with $g_{n}(x) \rightarrow 0$ a.e. we have $\left\|T g_{n}\right\|_{\infty} \rightarrow 0$.

(iii) $T$ is order continuous and for every disjoint sequence $g_{n} \in L_{\rho}$ with $0<g_{n}<g$ $\in L_{\rho}$ we have $\left\|T g_{n}\right\|_{\infty} \rightarrow 0$.

(iv) $T$ is order continuous and for every disjoint sequence $g_{n} \in L_{\rho}$ with $0<g_{n}<g$ $\in L_{\rho}$ we have $\left\||T| g_{n}\right\|_{\infty} \rightarrow 0$.

Proof. (i) $\Rightarrow$ (ii) Let $0 \leqslant g_{n} \leqslant g \in L_{\rho}$ with $g_{n} \rightarrow 0$ a.e. Then $A_{\lambda}=\left\{f \in L_{\rho}:|f|\right.$ $\leqslant c \cdot g\}$ is a Banach function space with respect to the norm

$$
\lambda(f)=\inf (c:|f| \leqslant c \cdot g) .
$$


We observe also that $A_{\lambda}^{\prime}$ can be identified with $L_{1}(g d \nu)$. Then $T$ considered as an operator from $A_{\lambda}$ into $L_{\infty}$ is by assumption weakly compact. Hence $T^{*}: L_{\infty}^{*} \rightarrow A_{\lambda}^{*}$ is $\sigma\left(A_{\lambda}^{*}, A_{\lambda}^{* *}\right)$ compact. From the order continuity of $T$ it follows that $T^{*}\left(L_{1}\right) \subset A_{\lambda}^{\prime}$ $=L_{1}(g d \nu)$. Denote by $T^{\prime}$ the restriction of $T^{*}$ to $L_{1}(X, \mu)$. Then $T^{\prime}: L_{1}(X, \mu) \rightarrow$ $L_{1}(g d \nu)$ is weakly compact. It follows now from Remark 2, following Theorem 1.1., that

$$
\sup \left(\int\left|T^{\prime} f\right| \cdot g_{n} d \nu:\|f\|_{1} \leqslant 1\right) \rightarrow 0
$$

so also

$$
\sup \left(\left|\int T^{\prime} f \cdot g_{n} d \nu\right|:\|f\|_{1} \leqslant 1\right) \rightarrow 0
$$

i.e., $\left\|T g_{n}\right\|_{\infty} \rightarrow 0$.

(ii) $\Rightarrow$ (iii) Obvious.

(iii) $\Rightarrow$ (iv) Assume (iv) is false. Then there exist $\varepsilon>0,0 \leqslant g_{n} \leqslant g \in L_{\rho}$ with $g_{n} \wedge g_{m}=0$ for $n \neq m$ such that $\left\||T| g_{n}\right\|_{\infty}>\varepsilon$. From the formula $|T| g_{n}=$ $\sup \left(|T f|:|f| \leqslant g_{n}\right)$ we conclude that

$$
\left\||T| g_{n}\right\|_{\infty}=\sup \left(\|T f\|_{\infty}:|f| \leqslant g_{n}\right) \text {. }
$$

Hence for all $n$ there exist $f_{n} \in L_{\rho},\left|f_{n}\right| \leqslant g_{n}$ such that $\left\|T f_{n}\right\|_{\infty}>\left\||T| g_{n}\right\|-\varepsilon / 2$, so $\left\|T f_{n}\right\|_{\infty} \geqslant \varepsilon / 2$ for all $n$. By applying (iii) to the sequence $\left\{f_{1}^{+}, f_{1}^{-}, f_{2}^{+}, f_{2}^{-}, \ldots\right\}$ we get a contradiction.

(iv) $\Rightarrow$ (i) Let $0 \leqslant g \in L_{\rho}$ and, as in the proof of (i) $\Rightarrow$ (ii), denote by $A_{\lambda}$ the Banach function space $\left\{f \in L_{\rho}:|f| \leqslant c \cdot g\right\}$ with the $A M$-norm $\lambda$. Then $|T|^{*}$ can be considered as a mapping from $L_{\infty}^{*}$ into $A_{\lambda}^{*}$ and $|T|^{\prime}$ denotes its restriction to $L_{1}(X, \mu)$. Then $|T|^{\prime}: L_{1}(X, \mu) \rightarrow A_{\lambda}^{\prime} \cong L_{1}(g d \nu)$. For $h \in A_{\lambda}$ we define the seminorm $\lambda_{1}$ by

$$
\lambda_{1}(h)=\sup \left(\int|T|^{\prime} f \cdot|h| d \nu:\|f\|_{1} \leqslant 1\right) .
$$

From (iv) it follows that $\lambda_{1}$ has the property that if $0 \leqslant h_{n} \leqslant h \in A_{\lambda}, h_{n} \wedge h_{m}=0$ for $n \neq m$, then $\lambda_{1}\left(h_{n}\right) \rightarrow 0$. It follows now from Meyer-Nieberg's result on disjoint sequences (see [9, Chapter II]) that $\lambda_{1}$ is order continuous, i.e. if $f_{n} \in A_{\lambda}, f_{n} \downarrow 0$ a.e., then $\lambda_{1}\left(f_{n}\right) \downarrow 0$. It follows from Theorem 1.1 that $\left\{|T|^{\prime} f:\|f\|_{1}<1\right\}$ is a $\sigma\left(A_{\lambda}^{\prime}, A_{\lambda}^{\prime \prime}\right)$ relatively compact subset of $A_{\lambda}^{\prime}$. If we denote by $T^{\prime}$ the restriction of $T^{*}$ to $L_{1}(X, \mu)$, then $\left|T^{\prime}\right| \leqslant|T|^{\prime}$ (actually $\left|T^{\prime}\right|=|T|^{\prime}$ here, but we do not need this stronger result). Hence, $\left\{T^{\prime} f:\|f\|_{1} \leqslant 1\right\}$ is also a $\sigma\left(A_{\lambda}^{\prime}, A_{\lambda}^{\prime \prime}\right)$ relatively compact subset of $A_{\lambda}^{\prime}$, i.e., $T^{\prime}: L_{1} \rightarrow A_{\lambda}^{\prime}$ is weakly compact. It follows that $\left(T^{\prime}\right)^{*}: A_{\lambda}^{\prime \prime}=A_{\lambda}$ $\rightarrow L_{\infty}(X, \mu)$ is weakly compact, i.e., $T[0, f]$ is relatively weakly compact in $L_{\infty}(X, \mu)$. This completes the proof of the theorem.

REMARK. The equivalence (i) $\Leftrightarrow$ (iii) could have been proved using some results of Grothendieck (see [8, Lemma 3.1]). The above result could also partly be derived from Theorem 4.2 of [2]. Moreover, from Theorem 6.4 of [2] it follows that the set of operators satisfying (i)-(iv) is strongly closed. 
THEOREM 2.6. Let $L_{\rho}$ be a Banach function space and let $T: L_{\rho} \rightarrow L_{\infty}$ be a norm bounded linear operator satisfying one of the four equivalent conditions of Theorem 2.5. Then $T$ is a kernel operator.

Proof. Condition (ii) implies, by Theorem 2.1 , immediately that $T$ is a kernel operator.

THEOREM 2.7. Let $L_{\rho}$ be a Banach function space. Then the set of all bounded operators from $L_{\rho}$ into $L_{\infty}(X, \mu)$ satisying one of the four conditions of Theorem 2.5 is an order ideal in $\mathcal{L}_{b}\left(L_{\rho}, L_{\infty}\right)$.

Proof. Immediate from Theorem 2.5.

COROLlARY 2.8. The set of order continuous weakly compact operators from $L_{\infty}(Y, \nu)$ into $L_{\infty}(X, \mu)$ is an order ideal in $\varrho_{b}\left(L_{\infty}, L_{\infty}\right)$, consisting of kernel operators.

ExAmple. Let $Y=\mathbf{N}, \nu$ counting measure and $X=[0,1], \mu$ Lebesgue measure. Let $g_{n}$ be the characteristic functon of the interval $\left(1 / 2^{n}, 1 / 2^{n-1}\right)$. Then $T: l_{\infty} \rightarrow$ $L_{\infty}[0,1]$ is defined by $T\left(\left\{\alpha_{n}\right\}\right)=\sum_{n=1}^{\infty} \alpha_{n} g_{n}$. Then $T$ is a positive kernel operator, but $T$ is not weakly compact, since $\left\|T\left(e_{n}\right)\right\|_{\infty}=1$ for all $n$, where $e_{n}=$ $(0, \ldots, 1,0, \ldots)$. This example shows that the weakly compact operators from $l_{\infty}$ into $L_{\infty}([0,1])$ do not form a band in $\mathcal{E}_{b}\left(l_{\infty}, L_{\infty}\right)$.

3. A characterization of integral operators in terms of equimeasurable sets. We begin with recalling a notion due to $\mathrm{A}$. Grothendieck.

Definition [3, 20]. A set $H \subset M(X, \mu)$ is called equimeasurable if for all $\varepsilon>0$ and all $X_{0} \subset X$ with $\mu\left(X_{0}\right)<\infty$ there exists $X_{1} \subset X_{0}$ with $\mu\left(X_{0} \backslash X_{1}\right)<\varepsilon$ such that $\left\{h \chi_{X_{1}}: h \in H\right\}$ is a relatively compact subset of $L_{\infty}(X, \mu)$.

We start with two lemmata.

Lemma 3.1. Let $\left\{g_{n}: n=1,2, \ldots\right\} \subset M(X, \mu)$ be equimeasurable and assume $g_{n} \stackrel{*}{\rightarrow} 0$. Then $g_{n}(x) \rightarrow 0$ a.e.

PROoF. The proof is straightforward and therefore omitted.

LEMMA 3.2. Let $T: L_{\infty}(Y, \nu) \rightarrow L_{\infty}(X, \mu)$ be an integral operator and assume $\mu(X)<\infty$. Then for all $\varepsilon>0$ there exists $X_{0} \subset X$ with $\mu\left(X \backslash X_{0}\right)<\varepsilon$ such that $P_{0} \circ T: L_{\infty}(Y, \nu) \rightarrow L_{\infty}(X, \mu)$ is compact, where $P_{0}$ denotes the operator $P_{0} f=$ $\chi_{X_{0}} f$.

Proof. First we observe that $T$ is norm bounded: A proof of this can be given by means of the closed graph theorem, similar to the proof for $L_{2}$-space as in [4]. Hence $T$ is order bounded, so if $T$ has $\operatorname{kernel} T(x, y)$, then $|T(x, y)|$ is the kernel of $|T|$ (see [6] and [10] for a simpler proof). It follows that $\iint|T(x, y)| d \nu(y) d \mu(x)<$ $\infty$. Hence there exist $t_{n}(x, y) \in L_{1}(X \times Y, \mu \times \nu)$ of the form $\sum_{1}^{m} a_{k} \chi_{A_{k}}(x) \chi_{B_{k}}(y)$ such that $t_{n}(x, y) \rightarrow T(x, y)$ in $L_{1}(X \times Y, \mu \times \nu)$. Passing to a subsequence we may assume that the convergence is also pointwise. From Fubini's theorem it follows that we may assume that $h_{n}(x)=\int\left|t_{n}(x, y)-T(x, y)\right| d \nu(y) \rightarrow 0$ a.e. on $X$. 
Let $\varepsilon>0$. Then, by Egoroff's theorem, there exists $X_{0} \subset X$ with $\mu\left(X \backslash X_{0}\right)<\varepsilon$ such that $h_{n}(x) \rightarrow 0$ uniformly on $X_{0}$. Define $S_{n}$ from $L_{\infty}(Y, \mu)$ into $L_{\infty}(X, \mu)$ by means of

$$
S_{n} f(x)=\int \chi_{x_{0}}(x) t_{n}(x, y) f(y) d \nu(y)
$$

Then $S_{n}$ is a finite rank operator and we shall show that $\left\|S_{n}-P_{0} \circ T\right\| \rightarrow 0$, where $P_{0}$ denotes the operator $P \circ f=\chi_{x_{0}} \cdot f$. Let $f \in L_{\infty}(Y, \nu)$ with $\|f\|_{\infty}<1$. Then

$$
\begin{aligned}
\left|S_{m} f(x)-P_{0} \circ T f(x)\right| & \leqslant \chi_{X_{0}}(x) \cdot \int\left|t_{m}(x, y)-T(x, y)\right||f(y)| d \nu(y) \\
& \leqslant \chi_{X_{0}}(x)\left(\int\left|t_{m}(x, y)-T(x, y)\right| d \nu(y)\right)\|f\|_{\infty} \leqslant \chi_{X_{0}}(x) h_{m}(x) .
\end{aligned}
$$

Hence

$$
\sup \left(\left\|S_{m} f-P_{0} \circ T f\right\|_{\infty}:\|f\|_{\infty} \leqslant 1\right) \leqslant\left\|\chi_{x_{0}} h_{m}\right\|_{\infty} \rightarrow 0
$$

as $m \rightarrow \infty$, i.e., $\left\|S_{m}-P_{0} \circ T\right\|_{\infty} \rightarrow 0$ as $m \rightarrow \infty$. We conclude from this that $P_{0} \circ T$ is compact.

REMARK. As noted by the referee, one could also prove the above theorem by some well-known vector measure theory. We outline the proof. Denote by $g_{x}$ the $L_{1}$-valued function $g_{x}(y)=T(x, y)$. Then $T f(x)=\left\langle g_{x}, f\right\rangle$. By a theorem of D. R. Lewis $\left[1\right.$, p. 88] we conclude, since $L_{1}$ is weakly compactly generated, that we may assume that $g_{x}$ is strongly measurable. The set $X_{0}$ is then found via the Egoroff theorem.

We note that the above lemma is, of course, false in case $\mu(X)=\infty$, since we can take as a counterexample the identity operator from $l_{\infty}$ into $l_{\infty}$. Let now $L$ and $M$ be ideals of measurable functions in $M(Y, \nu)$ and $M(X, \mu)$, respectively. Then a linear operator $T: L \rightarrow M$ is called *-continuous if it follows from $0<u_{n}<u \in L$ and $u_{n} \stackrel{*}{\rightarrow} 0$ that $T u_{n} \stackrel{*}{\rightarrow} 0$. With these notations we have the following theorem.

THEOREM 3.3. The linear operator $T: L \rightarrow M$ is an integral operator if and only if $T$ is *-continuous and maps order intervals into equimeasurable sets.

Proof. Let $T: L \rightarrow M$ be an integral operator. Then $T$ is certainly *-continuous (see Theorem 2.1). Let $0 \leqslant f \in L, X_{0} \subset X$ with $\mu\left(X_{0}\right)<\infty$ and $\varepsilon>0$. Then there exist $M>0$ and $X_{1} \subset X_{0}$ with $\mu\left(X_{0} \backslash X_{1}\right)<\varepsilon / 2$ such that $\int|T(x, y) f(y)| d \nu(y)<$ $M$ on $X_{1}$. Denote $A_{f}=\{g \in L:|g| \leqslant c f\}$ and by $P_{1}$ the operator $P_{1} f=\chi_{x_{1}} f$. Then $P_{1} \circ T$ is an integral operator from $A_{f}$ into $L_{\infty}\left(X_{1}, \mu\right)$. It follows from the above lemma that there exists $X_{2} \subset X_{1}$ with $\mu\left(X_{1} \backslash X_{2}\right)<\varepsilon / 2$ such that $P_{2} P_{1} T$ is a compact operator from $A_{f}$ into $L_{\infty}\left(X_{1}, \mu\right)$, where $P_{2}$ denotes the operator $P_{2} f=$ $\chi_{X_{2}} f$. Hence $\left\{\chi_{X_{2}} T g:|g| \leqslant f\right\}$ is a relatively compact subset of $L_{\infty}(X, \mu)$, and since $\mu\left(X_{0} \backslash X_{2}\right) \leqslant \varepsilon$ it follows that $T([-f, f])$ is an equimeasurable subset of $M(X, \mu)$. Assume now that $T$ is *-continuous and maps order intervals into equimeasurable sets. Let $0 \leqslant u_{n}<u$ in $L$ with $u_{n} \stackrel{*}{\rightarrow} 0$. Then by assumption $\left\{T u_{n}: n\right.$ $=1,2, \ldots\}$ is equimeasurable and $T u_{n} \stackrel{*}{\rightarrow} 0$. It follows now from Lemma 3.1 that $T u_{n}(x) \rightarrow 0$ and from Theorem 2.1 we conclude that $T$ is an integral operator. 
Corollary 3.4 (W. Schachermayer [7]). The linear operator $T: L_{p}(Y, \nu) \rightarrow$ $L_{q}(X, \mu)$, with $1 \leqslant p<\infty, 1 \leqslant q \leqslant \infty$, is an integral operator if and only if $T$ maps order intervals into equimeasurable sets.

Proof. If $T$ maps $L_{p}$ into $L_{q}$ and order intervals into equimeasurable sets, then a closed graph argument shows that $T$ is norm bounded (see [7]). The corollary now follows immediately, since norm bounded operators from $L_{p}(1<p<\infty)$ into $L_{q}$ are *-continuous.

REMARK. The above corollary was first observed by Schachermayer, who proved it by using vectorvalued integration.

We note another corollary of Theorem 3.3.

Corollary 3.5. Let $L$ be an ideal in $M(Y, \nu)$ and let $L_{\rho}=L_{\rho}(X, \mu)$ be a Banach function space. If $T: L \rightarrow L_{\rho}$ is an integral operator, then it follows from $0<u_{n}<u$ in $L$ and $\rho\left(T u_{n}\right) \rightarrow 0$ that $T u_{n}(x) \rightarrow 0$ a.e.

Proof. From $\rho\left(T u_{n}\right) \rightarrow 0$ it follows that $T u_{n} \stackrel{*}{\rightarrow} 0$. From Theorem 3.3 we know that $\left\{T u_{n}\right\}$ is equimeasurable, so by Lemma 3.1 we have $T u_{n}(x) \rightarrow 0$ a.e.

The converse of the above corollary is false in general, as can be seen by taking $L=L_{\rho}=L_{\infty}([0,1])$ and $T=$ the identity operator. There are, however, two partial converses, which follow immediately from Theorem 2.1.

(1) Let $L_{\rho_{1}}, L_{\rho_{2}}$ be Banach function spaces and assume $\rho_{2}$ is order continuous. If $T: L_{\rho_{2}} \rightarrow L_{\rho_{1}}$ is norm bounded and has the property that it follows from $0 \leqslant u_{n}<$ $u$ in $L_{\rho_{2}}$ and $\rho_{1}\left(T u_{n}\right) \rightarrow 0$ that $T u_{n}(x) \rightarrow 0$, then $T$ is an integral operator.

(2) Let $L$ be an ideal in $M(Y, \nu)$ and $L_{\rho}$ a Banach function space with order continuous norm. If $T: L \rightarrow L_{\rho}$ is an order bounded order continuous operator with the property that it follows from $0 \leqslant u_{n} \leqslant u$ in $L$ and $\rho\left(T u_{n}\right) \rightarrow 0$ that $T u_{n}(x) \rightarrow 0$ a.e., then $T$ is an absolute integral operator.

We now indicate the relation between Theorem 3.3 and results of Grothendieck and of Nagel and Schlotterbeck. The conclusions drawn from these results are of a less elementary nature than some of the results above. To distinguish integral operators in our sense and in the sense of Grothendieck, we call the latter $G$-integral operators. With this notation A. Grothendieck [3] proved the following result.

Theorem 3.6 (A. Grothendieck). Let $E$ be a Banach space with unit ball $B$ and $T: E \rightarrow L_{1}(X, \mu)$ be a bounded operator. Then

(1) $T$ is $G$-integral if and only if $T(B)$ is order bounded in $L_{1}$.

(2) $T$ is nuclear if and only if $T(B)$ is order bounded in $L_{1}$ and equimeasurable.

For a proof in case $\mu(X)<\infty$, see [1, pp. 258-259]. For the sake of convenience we only consider now the case $L_{2}(Y, \nu)$ and $L_{2}(X, \mu)$. Then the result of Corollary 3.4 for absolute integral operators (i.e. order bounded integral operators) can be phrased as follows. Let $T: L_{2}(Y, \nu) \rightarrow L_{2}(X, \mu)$ be an order bounded operator. Then $T$ is an absolute integral operator if and only if for all $0<f \in L_{2}(Y, \nu)$ and all $0 \leqslant g \in L_{2}(X, \mu)$ the mapping induced by $T$ from $A_{f}=\left\{g \in L_{2},|g|<c f\right\}$ 
into $L_{1}(X, g d \mu)$ is nuclear. This is precisely the result of Nagel and Schlotterbeck obtained in the context of Banach lattices (see [9, Chapter IV]). It is for this reason that one can consider Theorem 3.3 as a nonorder bounded extension of their results.

\section{REFERENCES}

1. J. Diestel and J. J. Uhl, Jr., Vector measures, Math. Surveys, no. 15, Amer. Math. Soc., Providence, R. I., 1977.

2. P. G. Dodds, 0-weakly compact mappings of Riesz spaces, Trans. Amer. Math. Soc. 214 (1975), 389-402.

3. A. Grothendieck, Produits tensoriels topologiques et espaces nucléaires, Mem. Amer. Math. Soc., no. $16,1955$.

4. P. R. Halmos and V. S. Sunder, Bounded integral operators on $L^{2}$ spaces, Ergebnisse der Math. und ihrer Grenzgebiete, Springer-Verlag, Berlin and New York, 1978.

5. W. A. J. Luxemburg, Banach function spaces, Thesis, Assen, Netherlands, 1955.

6. W. A. J. Luxemburg and A. C. Zaanen, The linear modulus of an order bounded linear transformation, Nederl. Akad. Wetensch. Proc. Ser. A. 33 (1971), 422-447.

7. W. Schachermayer, Integral operators on $L^{P}$-spaces. I, Indiana J. Math. (to appear).

8. H. H. Schaefer, On the 0-spectrum of order bounded operators, Math. Z. 154 (1977), 79-84.

9. ___ Banach lattices and positive operators, Springer-Verlag, Berlin and New York, 1974.

10. A. R. Schep, Kernel operators, Nederl. Akad. Wetensch. Proc. Ser. A 82(1) (1979), 39-53.

11. , Generalized Carleman operators, Nederl. Akad. Wetensch. Proc. Ser. A 83(1) (1980), 49-59.

12. A. C. Zaanen, Integration, North-Holland, Amsterdam, 1967.

Department of Mathematics, California Institute of Technology, Pasadena, California 91125 